\title{
A SURVEY OF USING DIRECTIONAL ANTENNAS IN AD HOC NETWORKS
}

\author{
Ramezanali Sadeghzadeh ${ }^{1}$, Neshat Varjavand Naseri ${ }^{2}$ and Afsane Saee ${ }^{3}$ \\ ${ }^{1}$ Faculty of Electrical and Computer Engineering, K.N. Toosi University of Technology, \\ Tehran, Iran \\ sadeghz@eetd.knut.ac.ir \\ ${ }^{2}$ Dept. of Electrical and Computer Engineering, Science and Research Branch, Islamic \\ Azad University, Tehran, Iran \\ nvarjavand@yahoo.com \\ ${ }^{2}$ Dept. of Electrical and Computer Engineering, Science and Research Branch, Islamic \\ Azad University, Tehran, Iran \\ afsanesaeedyahoo.com
}

\begin{abstract}
In this paper, we present a comprehensive overview on Ad hoc networking by directional antennas. Use of Directional antennas can largely reduce the interference, increase the spatial reuse and due to their longer range we can have routes with fewer hop for two distant nodes. However the main problem of using directional antennas in Ad hoc networks is due to the dynamic nature of the network. Neighbour discovery, maintenance the track of moving neighbours, exploitation of the benefit of long range and directional MAC protocols are the most challenging issues. We present three Directional MAC protocols and two combinational protocols and system which give solutions to MAC and Neighbour discovery and compare the throughput of them with 802.11 with omnidirectional antennas.
\end{abstract}

\section{KEYWORDS}

Wireless Network, Ad hoc Network, Directional Antenna, MAC Protocol, Neighbour Discovery

\section{INTRODUCTION}

A mobile Ad hoc network is a wireless network without fixed base stations or any wire line backbone infrastructure. The network topology is continuously changing due to frequent node movements. Such networks are very useful in military and other tactical applications such as emergency rescue, where cellular infrastructure is unavailable or unreliable.

Pervious researches in wireless Ad hoc networks typically assume the use of omnidirectional antennas at all nodes. As two nodes are communicating using a given channel, MAC protocols such as IEEE 802.11 require all that nodes in the vicinity to stay silent. With directional antennas, two pairs of nodes located in each other's vicinity may potentially communicate simultaneously, depending on the directions of transmission. This can increase spatial reuse of the wireless channel. In addition, the higher gain of directional antennas provides a longer range and allows a node to communicate with other nodes located far away, implying that messages could be delivered to the destination in fewer hops. Increased spatial reuse and longer ranges translate into 
International Journal on AdHoc Networking Systems (IJANS) Vol. 2, No. 4, October 2012

higher Ad hoc network capacity (more simultaneous transmissions and fewer hops), and longer ranges also provide richer connectivity. Directional antennas reduce interference by directing beamforms toward a desired direction as a mobile node with a directional antenna, could largely reduce the unwanted interference to nodes lying outside its directional pattern. Similarly, a node that is receiving a packet can eliminate the interfering signals from directions other than the signal source, thereby using directional antennas increases the metric signal to interference and noise ratio (SINR).

Further, since the spatial signature of the energy is reduced to a smaller area, chances of eavesdropping are reduced, and with "smart" antennas, steering of nulls allows suppression of unnecessary interference (such as jammers), however a directional transmission due to its greater transmission range, may potentially interfere with communications taking place far away in that direction and due to selective reception, deafness can be occurred.

Replacing an omnidirectional antenna by a directional one in an ad hoc network is not by itself sufficient to exploit the offered potential. The antenna system needs to be appropriately controlled by each layer of the ad hoc networking protocol stack. Such control includes pointing in the right direction at the right time for transmitting and receiving, controlling the transmit power in accordance with the antenna gains, etc. Further, mechanisms that were designed with omnidirectional communications in mind -for example, medium access, neighbor discovery and routing- have to be redesigned for directional antennas. Finally, modifications to such mechanisms interact with each other -for instance, medium access control may require knowledge of how to beam form for a particular neighbor discovered by the neighbor discovery mechanism.

We divided the researches into two categories: first the protocols which assume that an upper layer is aware of the neighbors of a node and is capable of supplying the transceiver profiles required to communicate to each of these neighbors. These protocols such as MDD-MAC, DMAC and MMAC consider the effect of directional antenna only on MAC layer. The other mechanisms are combinational and consider interact of MAC layer with other layers and modules, such as UDAAN, PMAC and Quorum-based ones.

\section{MAC PROTOCOLS}

In this section we study the protocols which only consider the effect of directional antenna in MAC layer. At first we introduce a simple protocol named MDD, in next step we introduce a directional MAC protocol based on DNAV (Directional Network Allocation Vector) and at the end of this part we introduce a change in DMAC to exploit the transmission range of directional antenna.

\subsection{MDD Protocol}

This protocol is similar to IEEE 802.11, adapted for use with directional transmission. The key feature that has been added in the adaptation is a mechanism for transmitting and receiving nodes to determine the directions of each other. The task of finding the sequence of nodes through which to route a packet to the intended destination is performed by the routing protocol.

The MAC is concerned with only the destination for that hop, which is one of its neighbors, as specified by the routing layer. Each node has $\mathrm{M}$ directional antennas with non-overlapping beam directions, so as to collectively span the entire plan. MDD- MAC protocol can switch any or all of $\mathrm{M}$ antennas to active or passive mode. Considering the mobility of network, a node isn't aware of the location of its neighbors. Any node that wishes to send a packet to a neighbor, sends an 
omnidirectional RTS addressed to destination. Idle nodes listen on all their antennas, which it means that receiving is at first omnidirectional. Destination notes the direction of source (the antenna that received maximum power of RTS: S-Direction) and responds by CTS Omni directionally (on all $\mathrm{M}$ antennas). After all, destination sets S-Direction antenna to active and all others to passive mode, waiting for data. If source receives CTS correctly, it estimates the direction of the destination (the antenna that received maximum power of CTS which is noted as D-Direction) and transmits data on D-Direction. Destination transmits ACK in response, on SDirection [1].

The evaluation of the throughput performance of an ad hoc network is done with 225 nodes lying on a $15 \times 15$ uniform grid. The simulation parameters are shown in Table 1 . The variation of the total throughput of the network with the total offered load is shown in figure 1 for different values of $\mathrm{M}$ (number of antennas in each node). For the same network and traffic conditions, the throughput is found to increase with $\mathrm{M}$. The peak throughput nearly doubles when $180^{\circ}$ directional antennas $(\mathrm{M}=2)$ are used in place of the omnidirectional ones $(\mathrm{M}=1)$. For higher $\mathrm{Ms}$, the incremental improvement of throughput is less pronounced. It's shown that mobility has negligible effects on throughput for speeds up to $3 \mathrm{~m} / \mathrm{s}$.

Table 1. Parameter and values used in simulations

\begin{tabular}{|l|c|}
\hline Parameter & Values used \\
\hline Grid size & $200 \mathrm{~m}$ \\
\hline Transmitter power & $50 \mathrm{dBm}$ \\
\hline Carrier sense threshold (ST) & $-70 \mathrm{dBm}$ \\
\hline Noise floor & $-90 \mathrm{dBm}$ \\
\hline Minimum SIR & $20 \mathrm{~dB}$ \\
\hline Packet size & $1000 \mathrm{bytes}$ \\
\hline Node speed & $3 \mathrm{~m} / \mathrm{s}$ \\
\hline Total bandwidth & $1 \mathrm{Mb} / \mathrm{sec}$ \\
\hline
\end{tabular}

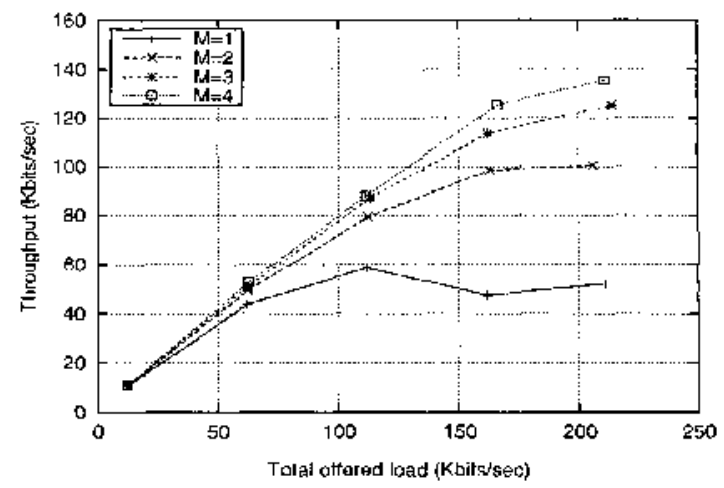

Figure 1. Throughput vs. Total offered load for an ad hoc network with 225 nodes lying on a $15 \times 15$ uniform grid, moving randomly with a speed of $3 \mathrm{~m} / \mathrm{s}$. 
International Journal on AdHoc Networking Systems (IJANS) Vol. 2, No. 4, October 2012

\subsection{DMAC Protocol}

In this protocol, we assume that an upper layer is aware of the neighbors of a node, and is capable of supplying the transceiver profilesfromupper layers along with the packet to be transmitted. Channel Reservation in DMAC is performed using RTS/CTS handshake, both being transmitted directionally. The antenna system has two seperate modes; Omni and Directional. Now we describe the DMAC protocol in three phase [2]:

a) RTS Transmission: Having received a packet and its transceiver profile $\left(\mathrm{T}^{\mathrm{p}}\right)$ from its upper layers, $\mathrm{D}$ requests the physical layer to beamform according to $\mathrm{T}^{\mathrm{p}}$. We denote this beamform by $B^{R}$, since this beam points in the direction of Receiving node $(R)$. Source node $(S)$ performs physical carrier sensing using $\mathrm{B}^{\mathrm{R}}$. If the channel is sensed idle, DMAC checks its Directional NAV (DNAV) to find out whether it must defer transmitting in the direction of node R. The DNAV table maintains a virtual carrier sense status for every Direction of Arrival (DoA) in which it has overheared a RTS or CTS packet for the duration time field in RTS/CTS. Once node $\mathrm{S}$ finds that it is safe to transmit using $\mathrm{B}^{\mathrm{R}}$, it enters the backoff phase. After backoff time, DMAC sends down RTS to the physical layer to be transmitted to node $R$, using beam $B^{R}$.

b) RTS Reception and CTS transmission: An Idle node listens Omnidirectionally. The antenna system is capable of determining the direction of arrival (DOA) of this incomming signal. Other nodes $(\mathrm{X})$ receiving RTS updates their DNAV table in the DoA. By receiving RTS, $\mathrm{R}$ determines the direction of node $\mathrm{S}\left(\mathrm{D}^{\mathrm{RS}}\right)$, then if the DNAV table permits transmission in $\mathrm{D}^{\mathrm{RS}}$, DMAC requests the physical layer to beamform in the direction of $\mathrm{B}^{\mathrm{S}}$. Physical layer at node $\mathrm{R}$ senses the channel using $\mathrm{B}^{\mathrm{S}}$, for SIFS time slots. If the channel remains free during this interval, CTS is transmitted using beam $\mathrm{B}^{\mathrm{S}}$.

c) CTS Reception and DATA/ACK Exchange: $\mathrm{S}$ waits for CTS in $\mathrm{B}^{\mathrm{R}}$, if CTS does not come back within a CTS-timeout, then S retransmit RTS. If S received CTS, it initiates the transmission of data using $\mathrm{B}^{\mathrm{R}}$. Node R responds by ACK in $\mathrm{B}^{\mathrm{S}}$. Other nodes update their DNAV table by receiving CTS, data or ACK.

DMAC protocol introduces two new Hidden terminal problems, one due to asymmetry in gain, and the other due to unheard RTS/CTS. The other problem is Deafness when two nodes want to send packet to the same node from an intermediate route. These problems don't permit to exploit the benefits of directional transmission. For the scenario shown in figure 2, Table 2 compares the throughput of links with 802.11.

The total throughput of DMAC is more than twice of 802.11 for 2(a) due to increase of spatial reuse. But 2(b) does not offer much benefit. Since the interfering range of directional antennas is larger, using DMAC, only one of the 3 transmissions can occur at any given time. Scenario 2(b) is also affected by the hidden terminal problem due to asymmetry gain, which happens because A neither receives F's CTS to C, nor senses C's data towards F. A would initiate directional transmission to D irrespective of whether $\mathrm{C}$ is communicating with $\mathrm{F}$. Thus even though a collision happens at $\mathrm{F}$, the packet from $\mathrm{A}$ to $\mathrm{D}$ is transmitted successfully. This prevents the contention window of node A growing exponentially, as a result A achieves a higher throughput. We'll study the other protocol MMAC which attempts to exploit the extended transmission range of directional antennas. 
International Journal on AdHoc Networking Systems (IJANS) Vol. 2, No. 4, October 2012

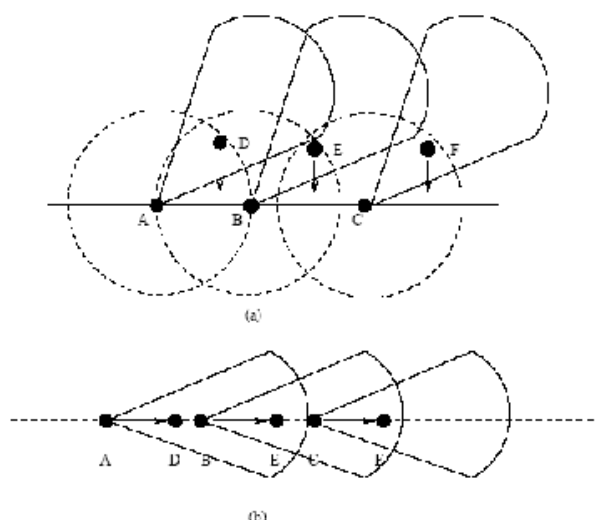

Figure 2. (a) A scenario allowing high spatial reuse for DMAC. (b) High directional interference and the hidden terminal problem degrade performance of DMAC.

Table 2. Comparing per flow throughput of DMAC and 802.11

\begin{tabular}{|c|c|c|}
\cline { 2 - 3 } \multicolumn{1}{c|}{} & \multicolumn{2}{c|}{ Per Flow Throughput (Kbps) } \\
\cline { 2 - 3 } \multicolumn{1}{c|}{} & IEEE 802.11 & Basic DMAC \\
\hline Figure 2(a) & & \\
\hline A to D & 409.05 & 1106.76 \\
\hline B to E & 379.92 & 628.82 \\
\hline C to F & 400.76 & 968.60 \\
\hline Aggregate & 1189.73 & 2704.18 \\
\hline \hline Figure 2(b) & & \\
\hline A to D & 391.54 & 978.66 \\
\hline B to E & 401.48 & 233.46 \\
\hline C to F & 401.79 & 207.39 \\
\hline Aggregate & 1194.81 & 1419.51 \\
\hline
\end{tabular}

\subsection{MMAC Protocol}

MMAC (Multi-Hop RTS MAC) protocol exploits the extended transmission range of directional antennas, while achieving spatial reuse comparable to the basic DMAC protocol. Although deafness and hidden terminal problems still exist in MMAC, better use of directional capabilities in MMAC can compensate for their negative impact, leading to improvement in performance. Referring to figure 3, if A transmits directionally, only B, G and D would be able to receive the signal while they are in their Omni mode. However, a communication may directly takes place between $\mathrm{A}$ and $\mathrm{F}$, if both are pointing their beams towards each other [2].

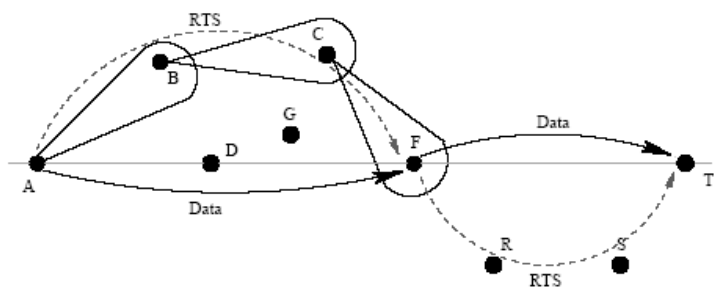

Figure 3. A scenario showing how the multi-hop RTS is forwarded from A to F 
International Journal on AdHoc Networking Systems (IJANS) Vol. 2, No. 4, October 2012

To describe the protocol we first define two kinds of neighbours: DO-neighbours and DD-neighbours.

- Direction-Omni (DO) neighbor: node B is a DO neighbor for node A if it can receive a directional transmission from $\mathrm{A}$ even if $\mathrm{B}$ is in Omni mode.

- Directional-Directional (DD) neighbor: node B is a DD neighbor for node A if it can receive a directional transmission from $\mathrm{A}$ only when $\mathrm{B}$ is beam formed in the direction of node $\mathrm{A}$ or through other nodes (DO-neighbor routes).

We assume that a module running above the MAC layer is capable of deciding the suitable DO-neighbour route to a DD-neighbour. Once the MAC layer received the packet with the route, the idea is to send a RTS along the DO- neighbour route to the DD-neighbour (destination) and request the destination node $(\mathrm{F})$ to point its receiving beam towards the RTS sender $(\mathrm{A})$ at a specific point of time in the near future.

The protocol has three phases:

a) RTS Transmission: MAC layer of node A receives a packet containing the DO-neighbor route to the destination DD-neighbor (A-B-C-F) and transceiver profiles: $\mathrm{T}^{\mathrm{B}}$ and $\mathrm{T}^{\mathrm{F}}$. MMAC checks if the physical layer is free. Then request to beamform in the direction $\mathrm{B}^{\mathrm{F}}$. MMAC sends RTS after physical carrier sensing, DNAV check and waiting for backofftime. Although the destination of this RTS is F but it may not reach that since F is listening Omnidirectionally. However the intention of sending this RTS in the direction of F is not to deliver the RTS to F , but to reserve the channel in the region between A and F. But if F happens to be beamformed in the direction of A, when A sends RTS, it can receive RTS and responds by CTS. If A receives CTS it initiates sending data to F. If the CTS does not arrive within timeout interval, node A constructs another RTS, a special one that is delivered to the destination over multiple hops (Forwarding RTS or Multi-Hop RTS) and contain DOneighbor route from A to F. This RTS is Transmitted hop by hop (along the DO- neighbor rute) until it's delivered to the destination. None of the nodes modify their DNAV on receiving or overhearing M-RTS. This RTS has the highest priority for transmission (intermediate nodes doesn't involve backing off).

b) RTS Reception and CTS transmission: On receiving the forwarding RTS, F points its beam in the direction of A. CTS is transmitted using beam $\mathrm{B}^{\mathrm{S}}$ after virtual and physical carrier sensing and wating for SIFS interval of time.

c) CTS Reception and DATA/ACK Exchange: A waits for CTS in $\mathrm{B}^{\mathrm{F}}$, if CTS does not come back within a CTS-timeout, then A retransmit RTS. If A received CTS, it initiates the transmission of data using $\mathrm{B}^{\mathrm{F}}$. Node $\mathrm{F}$ responds by ACK in $\mathrm{B}^{\mathrm{A}}$. Other nodes update their DNAV table by receiving CTS, data or ACK.

We do the simulation with 25 nodes with random topology and randomly selected routes, figure 4 compares the throughput of 802.11, DMAC and MMAC. Comparing with figure 1 , also it is obvious that DMAC and MMAC both have better performance compare to MDD protocol. At sending rates over $500 \mathrm{~kb} / \mathrm{s}$, the better performance of MMAC is more obvious. The simulations show that average end-to-end delay of MMAC is smaller than DMAC because MMAC requires only RTSs to travel on the DO-links and data to be transmitted on dd-links with fewer hops. However the higher failure probability in transmitting the multi-hop RTS in MMAC, increases the latency of packet delivery due to frequent time-outs and retransmissions. Therefore the performance of MMAC in terms of end-to-end delay is only slightly better in comparison to DMAC. 


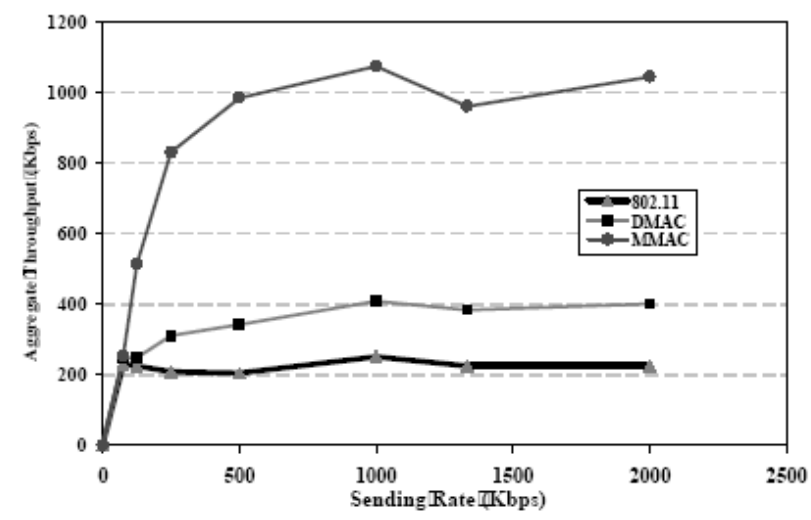

Figure 4. Average aggregate throughput of DMAC/MMAC over multiple simulations using random topologies and routes.

\section{Combinational protocols}

In this section we study the protocols which consider the effect of directional antenna in MAC and upper layers. At first we introduce a complete system named UDAAN then in next step we introduce a protocol based on polling for discovering the neighbours, PMAC.

\subsection{UDAAN}

It is the first such complete system for ad hoc networking using directional antennas, called UDAAN ("Utilizing Directional Antennas for Ad Hoc Networking"). While previous works have targeted specific problems, such as medium access for directional antennas, there has been no published work on designing, implementing and fielding a complete system that uses directional antennas. It presents novel mechanisms for medium access, neighbour discovery, link characterization and routing [3].

Due to space restrictions we do not describe the routing protocol, except to mention that routing is done by the Hazy-Sighted Link State protocol (HSLS), which is a proactive link state routing protocol. The individual modules use Linkprofiles to express the various modes of communicating with a neighbouring node. A linkprofile is a tuple of (band, beamform). Typically a single radio servicing a single "band" and a "beamform" indicates the antenna method used for exchanging packets as one of No Beamforming (NBF), Transmit Beamforming (T-BF), or Transmit and Receive Beamforming (TR-BF). The use of the term "beamforming" indicates the use of a directional antenna (either switched or steered). Therefore, "no beamforming" means that the transmitter and the receiver use omnidirectional antennas, while transmit beamforming means that the transmitter uses a directional antenna, but the receiver uses an omnidirectional antenna.

Two novel features of solution differentiate it from previous solution approaches like DMAC. The first is the use of a back off procedure where both the interval boundaries and the method of back off depend upon the event (e.g., no CTS, no ACK and channel busy) that caused the back off. The second is the tight integration of power control with direction control. The main idea of power control is that instead of using maximum transmission power, each node sets its power to a certain level. To increase fault tolerance of the network, nodes will consume more power. An optimum distance between the nodes of the network was calculated in [6] in order to reduce the power consumption of the nodes. The mentioned method assumes that all node antennas are omnidirectional although in next attempts we can consider the effect of using directional antennas instead of omnidirectional ones. Power control is critical to exploiting the potential of directional 
antennas. Thus, for instance, our DNAV table includes power values. The MAC protocol is designed to be simple enough to implement within a short time. Thus, rather than build in complex protocol features to eliminate all collisions, the protocol controls the collisions using judicious back off schemes to achieve high throughput in practice. When a node has a packet to send, it does directional carrier sensing (DCS) on the antenna corresponding to the destination for a randomly chosen period called the DCS-period. If the channel is free for the duration of the DCS-period, then the node sends an RTS. After the RTS is sent, the node listens on the same directional antenna and receives the CTS, sends the DATA and receives the ACK. All of this is done directionally.

The node goes into a forced idle if one of the following happens: the channel is busy, a CTS is not received and an ACK is not received. In forced idle the node is switched to the omni antenna and may receive packets, respond to RTS, etc. A forced idle period ends at the later of the completion of such communications and the expiration of a timer that is set when entering forced idle. A "contention window" is maintained and reset after each entry-exit sequence from the forced idle. The timer value is a random number within this contention window. As mentioned earlier, the way the contention window is reset depends upon the particular event. This is captured in table 3, thus, if the channel is busy, the node backs off, tries again (no change in the window size) and repeats this until a certain number of attempts is exceeded in which case the RTS is sent anyway. Similarly, if there is no CTS received, the node backs off using a "linear increase" method and if there is no ACK received, the node implements an "exponential increase" and "exponential decrease" of the contention window. After the ACK is received, the node goes into a forced idle to give other nodes a chance.

Table 3. FI modes and CW increase/decrease

\begin{tabular}{|l|l|}
\hline FI mode & Contention window \\
\hline FI- busy & Constant \\
\hline FI- No CTS & Linear increase \\
\hline FI- No ACK & Exponential increase \\
\hline FI- ACK & Exponential decrease \\
\hline
\end{tabular}

\section{Directional NAV and Power Control:}

The first RTS for a given packet is sent at the power indicated in the radio profile (or maximum power if none is specified). The RTS contains the power $P$ and its current receive threshold $(T)$. Then, the CTS is sent with a power equal to $P=(R-T)+M A R G I N-P O W E R$, where $\mathrm{R}$ is the received signal strength indication (RSSI) of the received RTS, and MARGIN-POWER is a margin to account for fades etc. The DATA and ACK are power-adjusted in a similar manner.

The NAV table contains, in addition to the duration field, the antenna number, and the allowed power. This field indicates the power above which interference will occur. It may be used to transmit if it is deemed (see below) that the intended transmission is sufficiently low power so as to not bother the busy nodes.

The allowed power field is set as follows. Only RTS/CTS are processed for NAV. When an RTS (CTS) is received, the allowed power is set as the smaller of the current allowed power and $P-(R-T)$-VCSMarginPower, where $P$ is the transmitted power of the RTS (CTS) (indicated in the RTS (CTS)), $R$ is the received power, $T$ is the current receive threshold of the sender of the RTS (CTS), and VCSMarginPower is a configured parameter to account for fades etc. 
When a packet is to be transmitted with a power $P_{x m i t}$ the following deference procedure is employed. If $P_{x m i t}$ is smaller than (allowedPower - $\left(G_{x m i t}-G_{o m n i}\right)$ ), then the packet is allowed to be transmitted, regardless of busy indication of the antenna. Here, $G_{x m i t}$ is the gain of the antenna that is intended to be used for transmission, and $\mathrm{G}_{\mathrm{omni}}$ is the gain of the omnidirectional antenna. This is required because the RTS and CTS are received omnidirectional. Otherwise, the node defers for a period indicated by the duration field in the NAV table.

\section{neighbor discovery:}

That is, the ability to discover neighbours that can only be reached if one or both of the nodes use beam forming. As mentioned earlier, UDAAN has three kinds of links/neighbours in each band: N-BF (without beam forming), T-BF (using transmit-only beam forming), and TR-BF (using transmit and receive beam forming). The hard problem in directional neighbour discovery, such as for T-BF and TR-BF linkprofiles, is in determining where to point, and when to point the antenna for transmit and/or receive. There are two methods: informed discovery and blind discovery. In informed discovery, a node has available some form of information about a nonneighbour (for instance, from the routing information) that will enable pointing. In blind discovery, a node _ is not even aware of the existence of another node. Blind discovery for $\mathrm{T}-\mathrm{BF}$ and TR-BF links is, however, far more challenging, yet the only approach when the network is disconnected, and we need to create links across the partition(s).

UDAAN neighbour discovery is based on sending and scoring heartbeats, which are periodic control messages broadcast by each node. For informed T-BF neighbour discovery, the heartbeats are sent directionally toward a potential neighbour. Here we describe the main ideas behind the novel blind TR-BF mechanism.

The main challenge with blind TR-BF discovery is to get two nodes that do not know of each other's existence to beam form toward each other simultaneously. To accomplish this, we require that the clocks on all of the nodes are synchronized with each other, as might be the case if the common clock source is GPS. Periodically, all nodes engaged in blind TR-BF discovery do the following (at the same time). A direction is chosen based on the time - imagine one of the hands of an analogue clock. Each node alternates randomly between sending heartbeats in that direction and listening in the opposite direction for such heartbeats. For example, at a certain point in time that corresponds to 1 o'clock in the imaginary analogue clock, all nodes point toward 30 degrees east of due north to send heartbeats and 30 degrees west of due south to receive heartbeats. As seen in Figure 5, for any two nodes, when the direction matches the angle between the nodes, this scheme will cause the transmit beam forming of one node to align with the receive beam forming of the other create an opportunity for the nodes to transfer a heartbeat. It is easy to see that after only one such cycle all TRBF neighbours that are within range will be discovered. In contrast to randomly scanning, this is a remarkable improvement without any more complexity, other than the requirement of approximately synchronized time.

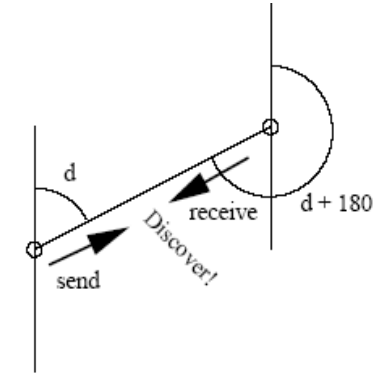

Figure 5. Blind TR-BF discovery 


\section{Link Characterization:}

The Link Characterization (Linkchar) module is designed to take information from the link layer interface and summarize it into a set of metrics for use by other modules. The minimum required energy for transmitting over a link is a very useful metric because it gives insight into the error rate, stability, and the probability of detection when using that particular linkprofile. Because of this, the routing module uses a quantized version of this energy metric for each link when creating paths in the network (it does not necessarily use the shortest number of hops).

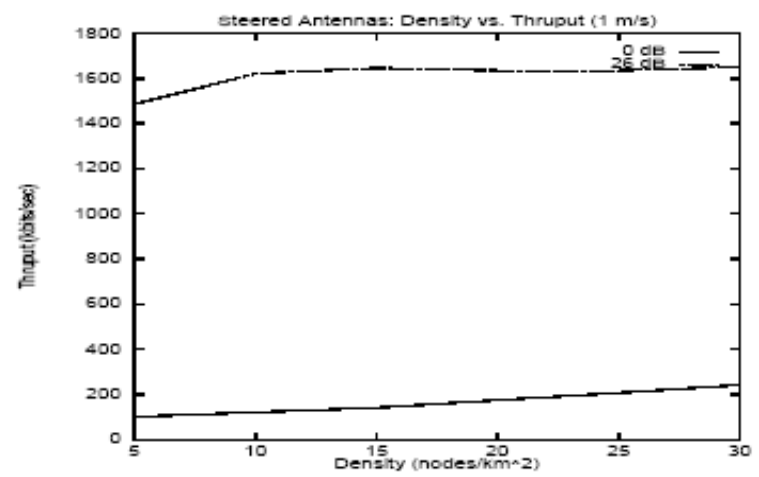

Figure 6. UDAAN throughput dependence on density

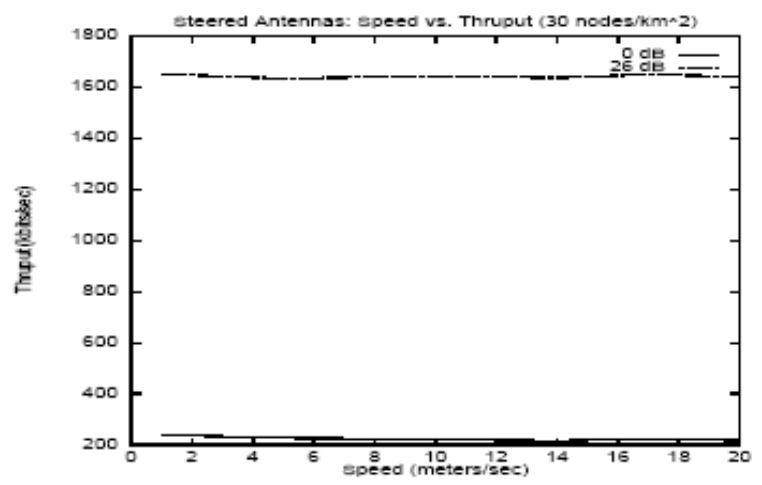

Figure 7. UDAAN throughput dependence on speed

This system is tested in practice and real world. For 20 nodes and sending rate of $400 \mathrm{~kb} / \mathrm{s}$, and antenna gain of $26 \mathrm{dbi}$, throughput was near $1600 \mathrm{~kb} / \mathrm{s}$. Comparing with DMAC and MMAC, (figure 4) the throughput of UDAAN in the same traffic, is more than the throughput of DMAC (near $350 \mathrm{~kb} / \mathrm{s}$ ) and MMAC (near $1000 \mathrm{~Kb} / \mathrm{s}$ ). Figures 6 and 7 illustrate the throughput performance of UDAAN depending on density and speed of nodes respectively.

\subsection{PMAC}

Most of the previously proposed schemes restrict themselves to either only directional transmissions or only directional receptions. The inability of exclusively using directional antennas for both the transmission and reception of all MAC layer frames (control or data) results in two major problems: (a) the spatial re-use benefits are reduced due to the invocation of omnidirectional communications and (b) the use of omnidirectional receptions for certain packets 
International Journal on AdHoc Networking Systems (IJANS) Vol. 2, No. 4, October 2012

and directional receptions for others leads to an inherent asymmetry in range. This phenomenon can result in the hidden terminal problem and leads to a significant penalty in throughput.

A challenge associated with the exclusive deployment of directional antennas is that, due to the angular reduction in range in comparison to the omnidirectional case, it is important for a node to poll each of its neighbours periodically to ensure that the neighbour's motion is tracked. The MAC protocols proposed thus far either completely ignore mobility or use omnidirectional transmissions or receptions.

PMAC (Polling-based MAC protocol) exclusively uses directional antennas for the transmission and reception of all the frames. Furthermore, by using polling, the maintenance of links to the discovered neighbours until they are outside the possible radial range of the node, becomes possible. Polling is also used to schedule the transmissions and receptions of information. At the scheduled time, the transmitter and the receiver nodes point their antenna beams towards each other and carry on the communication exclusively in directional mode. PMAC uses a scheme in which time is divided into contiguous frames as shown in Figure 8. Therefore, it is essential that each node in the network be synchronized with its neighbours in time [4].

\section{The Frame Structure:}

The PMAC protocol will allow a node to exist in one of three states;

- search state: searches for new neighbors

- polling state : polls known neighbors

- data transfer state : information is actually transferred.

Each frame is divided into three segments: Search segment, Polling segment and Data transfer segment.

Search segment: In the search segment, each node searches for new neighbours. Each slot can be further divided to four sub-slots. In the first sub-slot, the node would randomly choose to transmit its pilot tone (or identifier) or choose to receive. Both the transmissions and receptions are directional. If the node chose to receive in the first sub-slot it would transmit its pilot tone in the second sub-slot and vice versa.

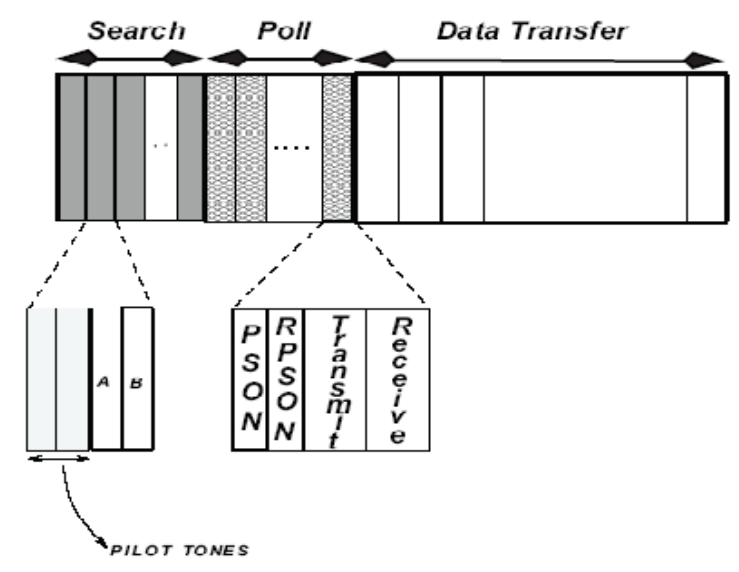

Figure 8. Frame structure in PMAC 
If there is a neighbour who has tuned his antenna in the same direction (in order to receive), when the node transmits, this neighbour will hear the pilot tone. Sub-slots 3 and 4 are labelled sub-slot $\mathrm{A}$ and sub-slot $\mathrm{B}$ in Figure 8. In sub-slots A and B, the nodes that successfully exchanged pilot tones, exchange $a$ list to specify the slots in their corresponding polling segments that are unused. The node that transmitted the pilot tone in the first sub-slot uses sub-slot A for transmitting its list; the other node of the pair transmits its list in sub-slot B. The two nodes with the help of each other's lists, then, identify a polling slot which can be used for scheduled polling.

Polling Segment: The polling slots serve twofold: first, they allow two nodes to re-establish contact periodically so that they can track each other and ensure that the link is maintained. Second, they can be used in order to schedule data transfers in the third part of the frame. Once, the nodes agree upon a polling slot, they communicate in the same slot periodically frame after frame until they cannot communicate with each other due to their moving out of each other's radial range. In a given frame, in the particular chosen polling slot, the nodes steer their antennas in the direction in which they had communicated with each other in the previous frame. Upon reestablishing the connection, the antenna directions are further tuned in order to maximize the signal strength with respect to each other. If the node were to transmit in the first part of the polling slot, it first takes into account its own data packet's transmission before accounting for the neighbour's transfer in the data transfer portion of the frame. If on the other hand, the node was the recipient in the first part of the polling slot, then it accounts for the transfer of its neighbour's data before accounting for its own. Accordingly, each node schedules the announced data transmission/reception in the data transfer part of the frame.

Data Transfer Segment: The scheduled data transfers take place in the data transfer portion of the frame. If a given node has a data transfer scheduled with a neighbour at a particular time (in a previous polling slot), the node points its antenna in the direction of the neighbour. Similarly, the neighbour would have pointed its antenna in the direction of the node under discussion. The scheduled packets are then transferred. An RTS and CTS message are included prior to the data transfer in order to detect possible rare collisions. PMAC either completely eliminate or alleviate the problems that are present in other previously proposed schemes[4].

This scheme can eliminate the Problems due to Range Asymmetry. As mentioned earlier, since this protocol uses only directional transmissions it avoids the problem of asymmetry in gain. It can also handle the mobility. Previous schemes either completely ignore mobility or use omnidirectional transmissions in order to detect neighbours that move out of angular range. In this scheme since PMAC polls neighbours periodically, we ensure that each node is continuously aware of its neighbours' positions. Even in the presence of burst traffic wherein a node may not exchange data with a neighbour for extended periods in time, the polling of the neighbour helps the node track the neighbour[5].

The other advantage of PMAC protocol is reducing the effects of deafness. Many of the previously proposed schemes suffer from the problem of deafness. As we know, when two nodes exchange control messages (RTS and CTS) directionally, a different neighbour of one of these communicating nodes may not hear the directional exchange. Later, during the data exchange between the nodes, this neighbour, being unaware of the data exchange might attempt to initiate communications with one of these nodes. However, clearly, it would not receive a response. This effect is referred to as deafness. As a consequence of the effects described, the neighbour would then back-off. The problem could repeat itself and may lead to the incorrectly concluding that a link failure has occurred. Since, our protocol is based on scheduled communications as opposed to asynchronous random access based communications, deafness does not occur.

The specific number of polling slots is an important parameter. In prior work on topology control in wireless networks, the number of neighbours and/or the power level to be used for such 
communications is computed for optimizing performance metrics such as connectivity. Takagi and Kleinrock in their classic work [7] have computed the number of neighbours that a node needs so that the progress of the packets towards their destinations is optimized (remains connectivity). That number was computed to be eight. The frame duration is a system parameter that should be appropriately chosen based on the expected mobility patterns. If nodes move with high speeds then the frame size should be small since, a node should poll its neighbours frequently in order to keep track of their positions. For simulation, we choose a speed of $2 \mathrm{~m} / \mathrm{s}$ to represent a pedestrian environment and a speed of $10 \mathrm{~m} / \mathrm{s}$ to represent vehicular environment. Some of the simulation parameters are shown in table 4.

Table 4. Simulation's parameters

\begin{tabular}{|l|l|}
\hline Search Segment Length (SSL) & 20 (slots) \\
\hline Poll Segment Length (PSL) & 8 (slots) \\
\hline Data Transfer Segment Length (DTSL) & 800 (slots) \\
\hline Packet size in slots 1,2 in Search Segment & 10 bytes \\
\hline Packet size in slots A,B in Search Segment & 20 bytes \\
\hline Poll packet size & 20 bytes \\
\hline Poll reply packet size & 14 bytes \\
\hline PSON packet size & 20 bytes \\
\hline RPSON packet size & 14 bytes \\
\hline RTS packet size & 20 bytes \\
\hline CTS packet size & 14 bytes \\
\hline Data packet size & 512 bytes \\
\hline Frame size & 1.64 seconds \\
\hline Data Transmission Rate & $2 \mathrm{Mbps}$ \\
\hline Channel frequency & $2.4 \mathrm{GHz}$ \\
\hline Antenna gain & $20 \mathrm{~dB}$ \\
\hline
\end{tabular}

As shown in Figure 9, with an increase in SSL, the number of frames that is required in order for the node to discover all of its neighbours decreases. When SSL $=20$, the node at the centre takes, on average, 5 frames to find all of its neighbours by using a $90 \pm$ antenna beam. If the antenna beam is $60 \pm$, the node takes about 10.5 frame durations on average. Clearly, the smaller the beam width, the higher the delay incurred in the initial discovery process. As an example, with a $30 \pm$ antenna beam, when SSL $=6$, the required number of frames is more than 100 . Note that with a 1.64 second frame size, this translates to less than 3 minutes. This might be acceptable since this search process is only required during the initialization phase.

Figure 10 compares the throughput of PMAC with 802.11 Vs. Network traffic. We want to compare PMAC throughput with MMAC, it is obvious that for traffic load of 1000 packets/s which is equal to $1000 \times 8 \times 512$ nearly $4000 \mathrm{~Kb} / \mathrm{s}$, throughput of PMAC is near $1800 \times 512 \times 8$ $(\approx 7000 \mathrm{~Kb} / \mathrm{s})$. Figure 4 shows that for this load, throughput is certainly near $1000 \mathrm{~kb} / \mathrm{s}$.

This protocol has the advantage of eliminating the asymmetry-in-gain problem but it requires netork synchronization. Furthermore, the optimal frame duration is a system parameter that may be difficult to obtain in dynamic networks. The neighbor discovery time is proportional to the number of antenna sectors. Finally, polling all of the one-hop neighbors may not be efficient hen the traffic is bursty[5]. 
International Journal on AdHoc Networking Systems (IJANS) Vol. 2, No. 4, October 2012

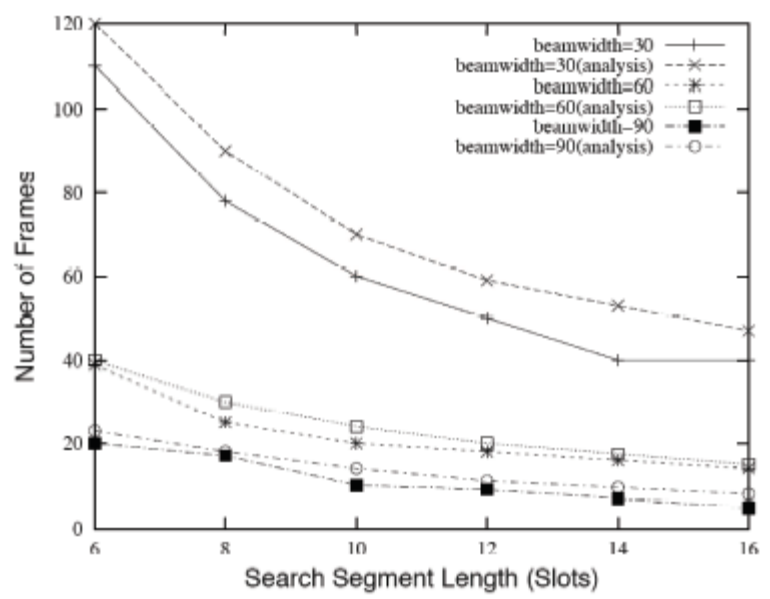

Figure 9. Number of frames required for centre node to find all possible neighbours in star topology.

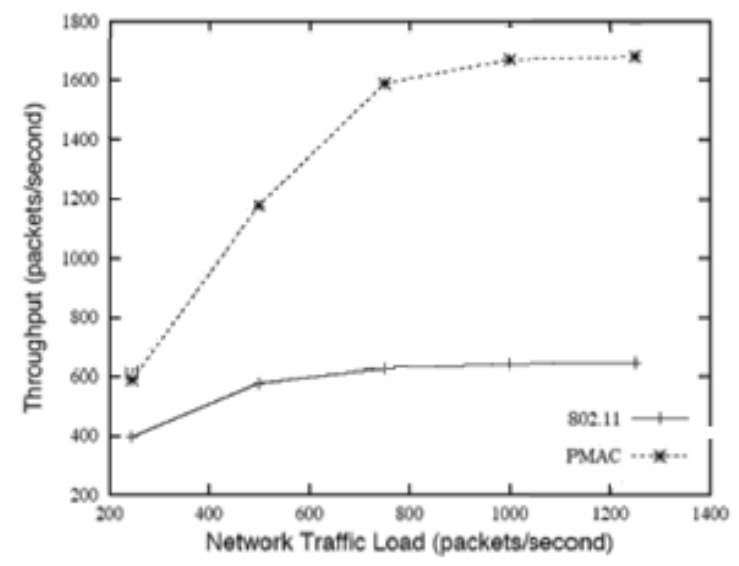

Figure 10. Total network throughput vs. network traffic load in stationary random topology

\subsection{Quorum-based Protocol}

In this protocol time is divided into equal intervals named: Advertise Interval. Each node activates its beam in 1-2 intervals. For directional antennas Neighbour nodes need to activate their beams (the beams towards each other) in common intervals. Each interval has two segments: Advertise window and Active window (Figure 11). These systems can work on either synchrony or asynchrony networks. All nodes try to send Advertise message which contains transmitting power and node ID after physical carrier sensing and back off time [7].

The receiver responds by ACK which contains transmitter and receiver ID in the advertise window. Scheduling for future communication is performed on Active window. There are many kinds of Quorum systems such as Grid quorum (figure 12) and Torus Quorum (figure 13). A Quorum shows the intervals which a beam is activated. In grid Quorum, if the antenna has K sectors, the grid must be $\mathrm{K}^{2}$ in dimension. Torus Quorum has $0.5 \mathrm{~K}^{2}$ rows and columns so search time is half of the grid system [8]. 
International Journal on AdHoc Networking Systems (IJANS) Vol. 2, No. 4, October 2012

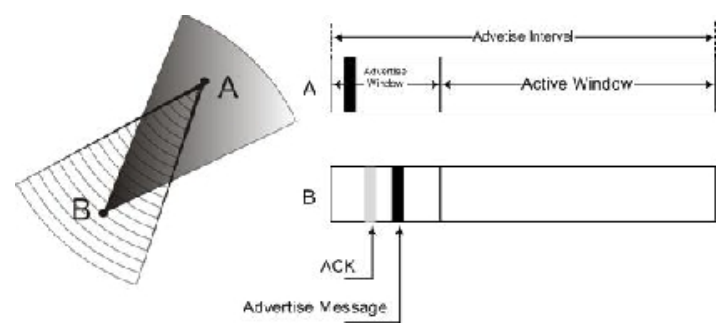

Figure 11: Advertise interval

\begin{tabular}{|c|c|c|c|}
\hline 0 & 1 & 2 & 3 \\
\hline 4 & 5 & 6 & 7 \\
\hline 8 & 9 & 10 & 11 \\
\hline 12 & 13 & 14 & 15 \\
\hline
\end{tabular}

\begin{tabular}{|c|c|c|c|}
\hline 0 & 1 & 2 & 3 \\
\hline 4 & 5 & 6 & 7 \\
\hline 8 & 9 & 10 & 11 \\
\hline 12 & 13 & 14 & 15 \\
\hline
\end{tabular}
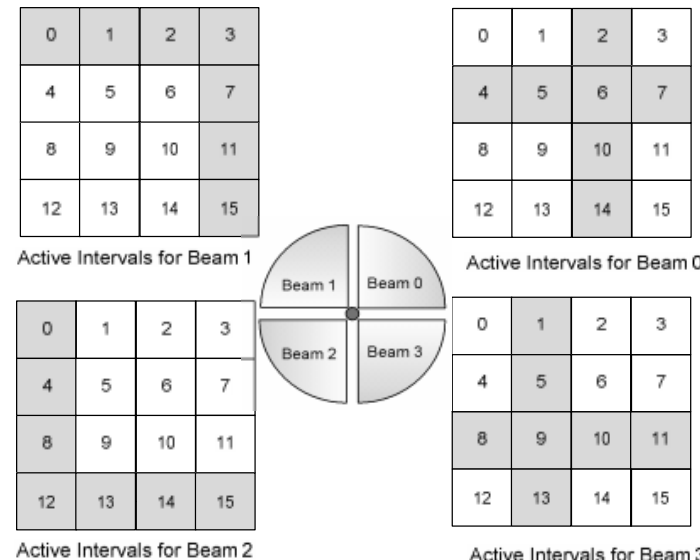

Active Intervals for Beam 0

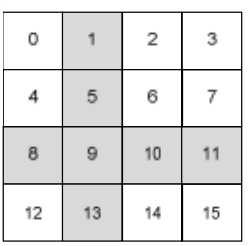

Active Intervals for Beam 2

Active Intervals for Beam 3

Figure 12. Grid Quorum

\begin{tabular}{|c|c|c|c|c|c|}
\hline 0 & 1 & 2 & 3 & 4 & 5 \\
\hline 6 & 7 & B & 9 & 10 & 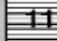 \\
\hline 12 & 13 & 74 & 15 & 16 & $=$ \\
\hline
\end{tabular}

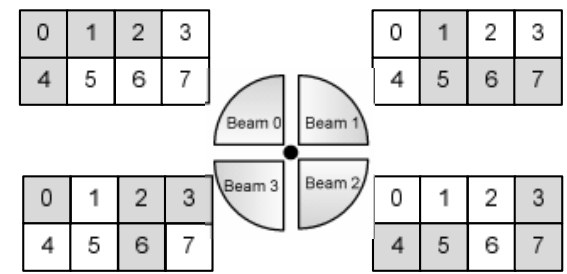

Figure 13. Torus Quorum

\section{Conclusions}

We proposed three MAC protocols which are adapted for directional transmission, and three combinational systems containing MAC and neighbour discovery. By MDD and DMAC we get an improvement in throughput compared to 802.11, but we didn't exploit fully of the benefits of directional transmission. By MMAC we exploit the longer range of directional antennas and make a direct link between two distant nodes, so improve the throughput. By UDAAN we proposed a practical system in real world which proposed a novel back off procedure to MAC and a unique way for blind neighbour discovery. PMAC was proposed. It is the first exclusive directional transmission and because of considering movement of nodes, is a great improvement in ad hoc directional transmission. Data transmission is scheduled so deafness can be cancelled. To this point PMAC has the best throughput. Quorum- based protocols are the most new ones proposed, they can be used in asynchronous networks. We can define quorums to improve the throughput of system. 
International Journal on AdHoc Networking Systems (IJANS) Vol. 2, No. 4, October 2012

\section{ACKNOWLEDGEMENTS}

The authors would like to thank Dr. M.A. Pourmina for his great guidelines.

\section{REFERENCES}

[1] A. Nasipuri, S. Ye, J. You \& R. Hiromoto, (2000) "A MAC Protocol for Mobile Ad hoc Networks Using Directional Antennas,” Proc. IEEE WCNC, Vol. 3, pp1214-1219.

[2] R.R. Choudhury, X. Yang, R. Ramanathan \& N. Vaidya, (2002) "Using Directional Antennas for Medium Access Control in Ad Hoc Networks,"Proc. ACM MOBICOM, Atlanta, Georgia.

[3] S. Ramanathan, J. Redi, C. Santivanez, D. Wiggins \& S. Polit, (2003) "Ad Hoc Networking with Directional antennas: A Complete System Solution,” BBN Technical Report No. 8370, Cambridge.

[4] G. Jakllari, W. Luo \& S.V. Krishnamurthy, (2007) "An Integrated Neighbour Discovery and MAC Protocol for Ad Hoc Networks Using Directional Antennas," IEEE Trans. Wireless Commun., Vol. 6, No. 3.

[5] E. Shihab, L. Cai \& J. Pan, (2008) "A Distributed Directional-to-Directional MAC Protocol for Asynchronous Ad Hoc networks," IEEE Proceedings. GLOBECOM.

[6] R. K. Shial, K.Reddy \& K.L. Narayana, (2012) "An Optimal RPC Based Approach to Increase Fault Tolerance in wireless Ad -Hoc Network," International Journal on AdHoc Networking Systems (IJANS), Vol. 2, No. 2.

[7] H. Takagi \& L. Kleinrock, (1984) "Optimal transmission ranges for randomly distributed packet radio terminals,” IEEE Trans. Commun., Vol. 32, No.3, pp246-257.

[8] J. R. Jiang, Y. C. Tseng, C. H. Hsu \& T. H. Lai, (2005) "Quorum-Based Asynchronous Power-Saving Protocols for IEEE 802.11 Ad Hoc Networks,” Mobile Networks and Applications, Vol. 10, No.3, pp169-181. 